\title{
NOTE ON FORMAL PROPERTIES OF CERTAIN CONTINUED FRACTIONS ${ }^{1}$
}

\section{FRIEDRICH L. BAUER AND EVELYN FRANK}

1. Introduction. It is the purpose of this note to point out the connection between the work of Bauer [1 $]^{2}$ and Frank [3] on certain continued fractions. A matrix approach similar to that in [1] shows clearly that these are analogues of Stieltjes-type and Jacobi-type continued fractions. An example is given of an expansion used for numerical purposes in [1], which is closely related to the Euler expansion [2]. This is obtained by a factorization of the Frobenius matrix. Special cases of such expansions coincide with certain cases of the hypergeometric continued fraction of Frank [3]. There is also a connection shown between the expansions in $\$ 2$ and the extended Schur continued fraction [4].

2. Matrix approach. Let $\pi_{n}$ define the space of polynomials of degree $n$ with coefficient of the highest power equal to 1 . If one is given a polynomial $P(x) \in \pi_{n}$ and a polynomial $p_{n-1}(x) \in \pi_{n-1}$, then there are defined polynomials $p_{\mu}(x) \in \pi_{\mu} \quad(n-2 \geqq \mu \geqq 0)$ and polynomials $r_{\mu}(x) \in \pi_{\mu} \quad(n-1 \geqq \mu \geqq 0)$ by the euclidean algorithm for $P(x)$ $\equiv r_{n}(x)$ and $p_{n-1}(x)$, as follows:

$$
\begin{aligned}
& (2.1) \quad x p_{i}(x)-r_{i+1}(x)=q_{n-i} r_{i}(x), \quad i=n-1, n-2, \cdots, 0, \\
& (2.2) \quad r_{i}(x)-p_{i}(x)=e_{n-i} p_{i-1}(x), \quad\left(e_{n} \equiv 0\right), i=n-1, n-2, \cdots, 0 .
\end{aligned}
$$

Define the row-vectors

$$
\begin{aligned}
& \mathcal{P}=\left(p_{n-1}(x), p_{n-2}(x), \cdots, p_{0}(x)\right), \\
& \mathcal{Q}=\left(r_{n-1}(x), r_{n-2}(x), \cdots, r_{0}(x)\right) .
\end{aligned}
$$

Then the euclidean algorithm takes the form

$$
x \cdot P=R Q \bmod P(x),
$$

and

$$
\Re=\odot E,
$$

Presented to the Society, November 15, 1957; received by the editors September 3 , 1957.

1 The preparation of this paper was sponsored in part by the Office of Naval Research, U.S. Navy. Reproduction in whole or in part is permitted for any purpose of the United States Government.

2 Numbers in brackets refer to the bibliography at the end of the paper. 
where $Q$ and $E$ are the "Stieltjes-type" matrices

(2.7)

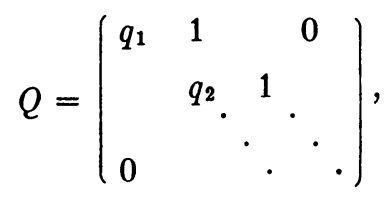$$
E=\left(\begin{array}{lllllll}
1 & & & & 0 & \\
e_{1} & 1 & & & \\
& e_{2} & 1 & & \\
& & \cdot & \cdot & \cdot & \\
0 & & & & & \cdot & 1
\end{array}\right) .
$$

On elimination of either $\mathcal{R}$ or $\mathcal{P}$, one obtains

$$
x \odot=\odot E Q \bmod P(x),
$$

and

$$
x R=R Q E \bmod P(x),
$$

where $E Q$ and $Q E$ are the tri-diagonal matrices

(2.11) $E Q=$

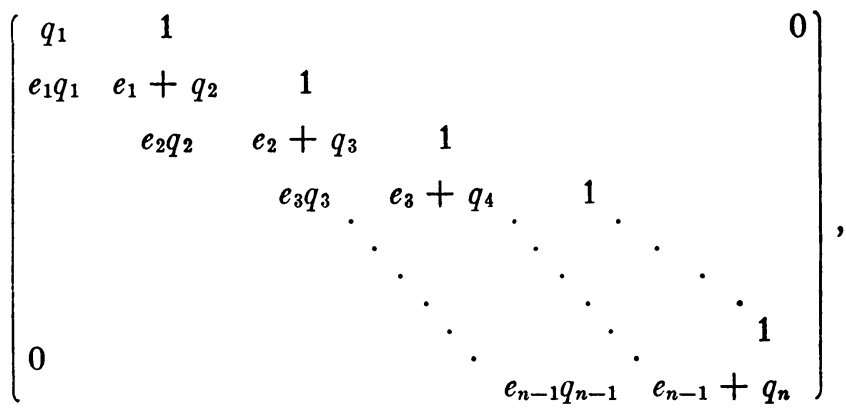

(2.12) $Q E=$

$$
\begin{aligned}
& e_{2} q_{3} \quad e_{3}+q_{3}
\end{aligned}
$$

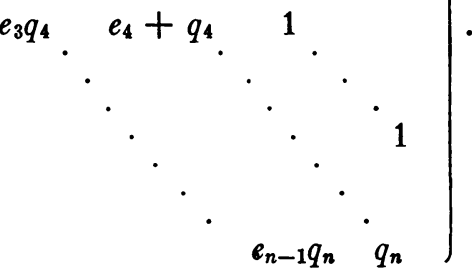


From these formulae one may write down immediately the two $J$ fractions

$$
\frac{p_{n-1}(x)}{P(x)}=\frac{1}{x-q_{1}}-\frac{e_{1} q_{1}}{x-q_{2}-e_{1}}-\cdots-\frac{e_{n-1} q_{n-1}}{x-q_{n}-e_{n-1}}
$$

and

$$
\frac{r_{n-1}(x)}{P(x)}=\frac{1}{x-q_{1}-e_{1}}-\frac{e_{1} q_{2}}{x-q_{2}-e_{2}}-\cdots-\frac{e_{n-1} q_{n}}{x-q_{n}}
$$

Furthermore, formulae (2.1) and (2.2) can be represented by the matrix equation

$$
(P R)\left(\begin{array}{lr}
x I & -E \\
-Q & I
\end{array}\right)=0 \bmod P(x) .
$$

The compound matrix in this formula may be rearranged to the tridiagonal form

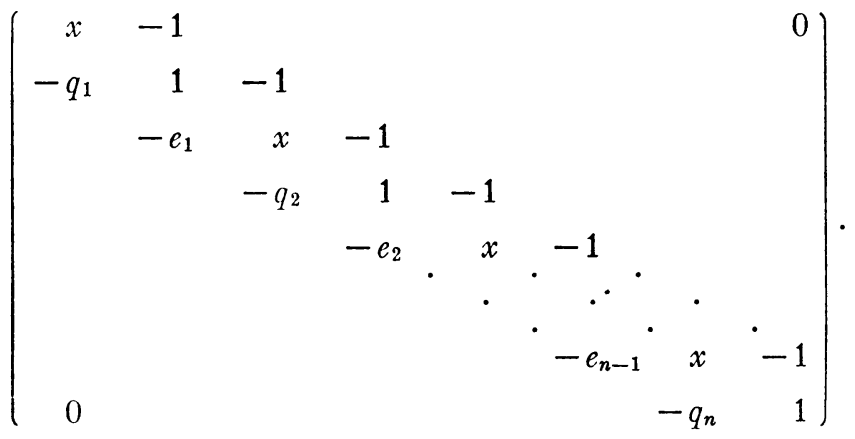

From this one obtains the Stieltjes continued fraction

$$
\frac{p_{n-1}(x)}{P(x)}=\frac{1}{x}-\frac{q_{1}}{1}-\frac{e_{1}}{x}-\cdots-\frac{q_{n}}{1} .
$$

2. A similar approach. It is possible to set up another division algorithm which differs from the preceding by the fact that the matrix $E$ in (2.6) is not a Stieltjes-type matrix but the reciprocal of such a matrix. In this algorithm, all quantities are denoted by an accent circonflexe. One has in this case

$$
x \cdot \widehat{P}=\widehat{\mathscr{R}} \hat{Q} \bmod P(x),
$$

$$
\widehat{R}=\widehat{P} \hat{E}^{-1} \text {, }
$$$$
x \cdot \widehat{P}=\widehat{\odot} \hat{E}^{-1} \hat{Q} \bmod P(x),
$$

$$
x \cdot \widehat{R}=\widehat{\mathscr{R}} \hat{Q} \hat{E}^{-1} \bmod P(x) \text {. }
$$


Formulae (2.5) and (2.6) give explicitly the division algorithm for $P(x) \equiv \hat{r}_{n}(x)$ and $\hat{r}_{n-1}(x)$, namely,

$$
\hat{r}_{i+1}(x)+\hat{q}_{n-i} \hat{p}_{i}(x)=x \hat{p}_{i}(x), \quad i=n-1, n-2, \cdots, 0,
$$

$$
\hat{p}_{i}(x)-\hat{r}_{i}(x)=\hat{e}_{n-i} \hat{\vartheta}_{i-1}(x),\left(\hat{e}_{n} \equiv 0\right), i=n-1, n-2, \cdots, 0 .
$$

From $(\widehat{2} .1)$ and $(\widehat{2} .2)$, or from $(\widehat{2} .10)$ in the form

$$
\widehat{R} \cdot(x \hat{E}-\hat{Q})=0 \bmod P(x),
$$

one obtains the analogue of (2.14),

$$
\frac{\hat{P}_{n-1}(x)}{P(x)}=\frac{1}{x-\hat{q}_{1}}+\frac{\hat{e}_{1} x}{x-\hat{q}_{2}}+\frac{\hat{e}_{2} x}{x-\hat{q}_{3}}+\cdots+\frac{\hat{e}_{n-1} x}{x-\hat{q}_{n}} .
$$

From $(\widehat{2} .1)$ and $(\widehat{2} .2)$ with $i$ replaced by $i+1$, one obtains the recurrence relation

$$
\begin{aligned}
\hat{p}_{i+1}(x)+\left(\hat{q}_{n-i}-\hat{e}_{n-i-1}\right) \hat{r}_{i}(x)= & x \hat{p}_{i}(x),\left(\hat{e}_{0} \equiv 0\right), \\
& i=n-1, n-2, \cdots, 0 .
\end{aligned}
$$

From $(\widehat{2} .1 \mathrm{a})$ and $(\widehat{2} .2)$ one can then derive the analogue of the Stieltjes-type continued fraction (2.16),

$$
\begin{aligned}
\frac{\hat{r}_{n-1}(x)}{P(x)} & =\frac{1}{-\hat{q}_{1}}+\frac{x}{1}-\frac{\hat{e}_{1}}{-\hat{q}_{2}+\hat{e}_{1}}+\frac{x}{1}-\frac{\hat{e}_{2}}{-\hat{q}_{3}+\hat{e}_{2}} \\
& +\frac{x}{1}-\cdots-\frac{\hat{e}_{n-1}}{-\hat{q}_{n}+\hat{e}_{n-1}}+\frac{x}{1}
\end{aligned}
$$

Furthermore, one can derive the analogue of the $J$-fraction (2.13)

$$
\begin{aligned}
\frac{\hat{p}_{n-1}(x)}{P(x)}= & \frac{1}{x-\frac{\hat{q}_{1}}{\hat{q}_{2}-\hat{e}_{1}} \hat{q}_{2}+x-\frac{\frac{\hat{q}_{1}}{\hat{q}_{2}-\hat{e}_{1}} \hat{e}_{1} x}{\hat{q}_{2}-\hat{e}_{1}} \hat{q}_{3}+x-\frac{\hat{q}_{2}-\hat{e}_{1}}{\hat{q}_{3}-\hat{e}_{2}}} \frac{\hat{e}_{2} x}{\hat{q}_{4}-\hat{e}_{2}} \hat{q}_{4}+\cdots \\
& \frac{\frac{\hat{q}_{n-2}-\hat{e}_{n-3}}{\hat{q}_{n-1}-\hat{e}_{n-2}} \hat{e}_{n-2} x}{\frac{\hat{q}_{n-1}-\hat{e}_{n-2}}{\hat{q}_{n}-\hat{e}_{n-1}} \hat{e}_{n-1} x} \\
+ & x-\frac{\hat{q}_{n-1}-\hat{e}_{n-2}}{\hat{q}_{n}-\hat{e}_{n-1}} q_{n}+x-\left(\hat{q}_{n}-\hat{e}_{n-1}\right)
\end{aligned}
$$

The continued fractions (2.13) and (2.14) are essentially the even and odd parts, respectively, of (2.16). Similarly, the analogues of these continued fractions, namely, $(\widehat{2} .13)$ and $(\widehat{2} .14)$, are the odd and even parts, respectively, of ( $\widehat{2} .16)$ (cf. Perron [5, pp. 12-13]). 
Note added in proof: For completeness we mention the following continued fraction

$$
\frac{\hat{p}_{n-1}(x)}{p(x)}=\frac{1}{x}-\frac{\hat{q}_{1} \hat{q}_{2}}{\hat{q}_{2}-\hat{e}_{1}}+\frac{\hat{e}_{1} x}{x}-\frac{\hat{q}_{2} \hat{q}_{3}}{\hat{q}_{3}-\hat{e}_{2}}+\frac{\hat{e}_{2} x}{x}-\ldots
$$

$(\hat{2} .13)$ and $(\hat{2} .14)$ are essentially the even and odd parts, respectively, of this continued fraction.

3. A continued fraction expansion for $1 /\left(1+a_{1} z+a_{2} z^{2}+a_{3} z^{3}+\cdots\right)$. The Frobenius matrix

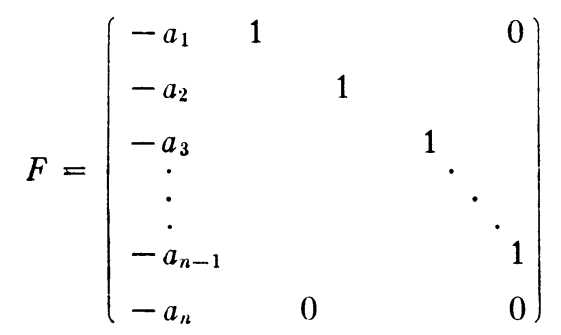

of the polynomial

$$
P(x)=x^{n}+a_{1} x^{n-1}+\cdots+a_{n-1} x+a_{n}
$$

can be factored into the product $\hat{E}^{-1} \hat{Q}$, namely,

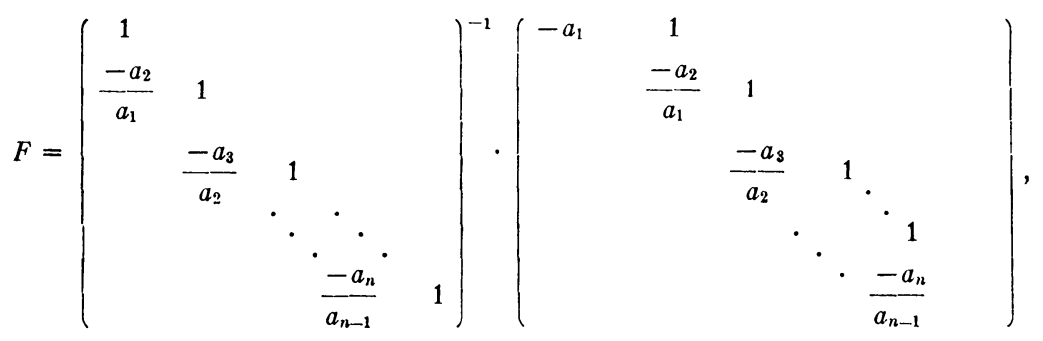

(cf. Bauer [1, p. 189]). Consequently, one can immediately write a special continued fraction of the form $(\widehat{2} .14)$ with $\hat{e}_{i}=-a_{i+1} / a_{i}$, $i=1,2, \cdots, n-1$, and $\hat{q}_{i}=-a_{i} / a_{i-1}, a_{0}=1, i=1,2, \cdots, n$. The polynomials $\hat{r}_{i}(x)$ from which this continued fraction is generated can be calculated from the recurrence formulae $(\hat{2} .1)$ and $(\hat{2} .2)$ where one starts with $r_{0} \equiv 1, p_{0} \equiv 1$. One obtains

$$
\hat{p}_{i}(x)=x^{i}, \quad i=0,1, \cdots, n-1,
$$

and

$$
\hat{P}_{i}(x)=\frac{1}{a_{n-i}}\left(a_{n-i} x^{i}+a_{n-i+1} x^{i-1}+\cdots+a_{n}\right)
$$


Therefore, the continued fraction expansion for $a_{1}^{-1}\left(P(x)-x^{n}\right) / P(x)$ is

$$
\frac{1}{x+a_{1}-x+\frac{a_{2}}{a_{1}} x}-\frac{\frac{a_{3}}{a_{2}} x}{x+\frac{a_{3}}{a_{2}}-\cdots-x+\frac{a_{n}}{a_{n-1}}},
$$

(formula 48 of Bauer [1]), or

$$
\frac{x^{n}}{P(x)}=1-\frac{a_{1}}{x+a_{1}-x+\frac{a_{2}}{a_{1}} x}-\cdots-\frac{\frac{a_{n}}{a_{1}} x}{x+\frac{a_{n}}{a_{n-1}}} .
$$

The substitution of $x=1 / z$ gives

$$
\begin{aligned}
& \frac{1}{1+a_{1} z+a_{2} z^{2}+\cdots+a_{n} z^{n}} \\
& =1-\frac{a_{1} z}{1+a_{1} z-1+\frac{a_{2}}{a_{1}} z}-\cdots-\frac{\frac{a_{n}}{a_{n-1}} z}{1+\frac{a_{n}}{a_{n-1}} z} .
\end{aligned}
$$

When one takes the reciprocal of both sides of (3.5) (even applied to an infinite sequence $\left\{a_{\mu}\right\}$ ), the resulting expansion is the well-known equivalent continued fraction of Euler [2] (with a slight transformation at the beginning),

$$
1+a_{1} z+a_{2} z^{2}+\cdots
$$

$$
\equiv \frac{1}{1-1+a_{1} z-1+\frac{a_{1} z}{a_{1}} z-1+\frac{a_{2}}{a_{1}} z} \frac{\frac{a_{3}}{a_{2}} z}{1+\frac{a_{3}}{a_{2}} z-\cdots} .
$$

If the series on the left-hand side of (3.6) converges, the equivalent continued fraction on the right-hand side converges to the same value, while, if the series diverges, the continued fraction likewise diverges.

4. Other special cases. For the special case of the hypergeometric function $F(\alpha, 1, \gamma, z),(3.6)$ takes the form 


$$
\frac{1}{F(\alpha, 1, \gamma, z)}=1-\frac{\frac{\alpha}{\gamma} z}{1+\frac{\alpha}{\gamma} z-1+\frac{\alpha+1}{\gamma+1} z-\cdots},
$$

valid for $|z|<1$ (cf. Frank [3, formula 5.2]). For $|z|>1$, (4.1) converges to the value 0 [3, Theorem 3.1].

Furthermore, expansions $(\widehat{2} .13),(\widehat{2} .14)$, and $(\widehat{2} .16)$ can be compared with the generalized Schur continued fractions (cf. Frank [4]). The expansion (1.1) of [4],

$$
\frac{k_{0}\left(1-\gamma_{0} \bar{\gamma}_{0}\right) z}{\bar{\gamma}_{0} z}-\frac{1}{k_{1} \gamma_{1}}+\frac{k_{1}\left(1-\gamma_{1} \bar{\gamma}_{1}\right) z}{\bar{\gamma}_{1} z}-\frac{1}{k_{2} \gamma_{2}+\cdots}, \quad, \quad\left|\gamma_{i}\right| \neq 1,
$$

is equivalent to the expansion (2.16) for $\hat{\vartheta}_{n-1}(1 / z) / P(1 / z)$ if the $k_{i}$ and $\gamma_{i}$ have the values

$$
\begin{aligned}
& k_{0}\left(1-\gamma_{0} \bar{\gamma}_{0}\right)=-1, \quad k_{i}\left(1-\gamma_{i} \bar{\gamma}_{i}\right)=\hat{e}_{i}, \\
& \bar{\gamma}_{0}=\hat{q}_{1}, \quad \bar{\gamma}_{i}=\hat{q}_{i+1}-\hat{e}_{i}, \quad i=1,2, \cdots, n-1,
\end{aligned}
$$

with the additional restriction

$$
k_{i} \gamma_{i}=1, \quad i=1,2, \cdots, n .
$$

With the same values (4.3) and (4.4), the even part of (4.2) (cf. expansion (6.1) of [4]) is equivalent to the expansion $(\hat{2} .14)$ for $\hat{\boldsymbol{r}}_{n-1}(1 / z) / P(1 / z)$. Also, the odd part of $(4.2)$ is equivalent with the values (4.3) and (4.4) to the expansion for $\hat{p}_{n-1}(1 / z) / P(1 / z)$ which can be obtained from the value for $\hat{p}_{n-1}(1 / z) / P(1 / z)$ from $(\hat{2} .13)$, as follows:

$$
\frac{\hat{r}_{n-1}(1 / z)}{P(1 / z)}=-\frac{1}{\hat{q}_{1}}+\frac{1}{\hat{q}_{1} z} \cdot \frac{\hat{p}_{n-1}(1 / z)}{P(1 / z)} .
$$

For these continued fractions, convergence regions are found in [4].

\section{REFERENCES}

1. Friedrich L. Bauer, Beiträge II, Direkte Faktorisierung eines Polynoms, Bayerische Akademie der Wissenschaften, Mathematisch-Naturwissenschaftliche Klasse, 1956, pp. 163-203.

2. L. Euler, De fractionibus continuis, Introductio in analysin infinitorum, Chapter 18, vol. 1, 1748 (Opera Omnia, Series Prima, vol. 8, pp. 362-390).

3. Evelyn Frank, A new class of continued fraction expansions for the ratios of hypergeometric functions, Trans. Amer. Math. Soc. vol. 81 (1956) pp. 453-476. 
4. - On the properties of certain continued fractions, Proc. Amer. Math. Soc. vol. 3 (1952) pp. 921-937.

5. Oskar Perron, Die Lehre von den Kettenbrüchen, vol. II, Stuttgart, Teubner, 1957.

Technische Hochschule München,

University of California at Los ANGeles and

UNIVERSITY OF ILLINOIS

\section{A THEOREM ON $A$-LOOPS}

\section{J. MARSHALL OSBORN}

In a recent article, R. H. Bruck and Lowell J. Paige have investigated $A$-loops, or loops for which every element of the inner mapping group is an automorphism (see [1]). Specifically, they have shown that $A$-loops with the inverse property are diassociative and that there exist noncommutative diassociative $A$-loops. The authors also conjecture that the only commutative diassociative $A$-loops are the commutative Moufang loops. The purpose of the present note is to offer a proof of this conjecture.

Let $y$ and $z$ be two elements of a commutative diassociative $A$-loop $G$, and let $R_{y}$ denote right multiplication in $G$ by the element $y$. Then $S=R_{z} R_{y} R_{z y}^{-1}$ is an element of the inner mapping group, so that $w S \cdot x S=(w x) S$ for every pair of elements $w$ and $x$ of $G$. Setting $w=p q$ and $x=q^{-1}$ gives $(p q) S=p S \cdot\left[q^{-1} S\right]^{-1}$, and comparing with the first equation, we see that

$$
\begin{aligned}
x R_{z} R_{y} R_{z y}^{-1} & =x S=\left[x^{-1} S\right]^{-1}=\left[\left(x^{-1} z \cdot y\right) \cdot(z y)^{-1}\right]^{-1} \\
& =\left(x z^{-1} \cdot y^{-1}\right) \cdot(z y)=x R_{z}^{-1} R_{y}^{-1} R_{z y} .
\end{aligned}
$$

Thus ${ }^{1} R_{z} R_{y} R_{z y}^{-1}=R_{z}^{-1} R_{y}^{-1} R_{z y}$, or $R_{z y}^{2}=R_{y} R_{z}^{2} R_{y}$. Using diassociativity, we can write this as $\left(x y \cdot z^{2}\right) \cdot y=x \cdot\left(y \cdot z^{2} y\right)$, which is just a form of the Moufang identity except for the fact that $z$ is squared. Our problem is to show that the identity holds without this restriction.

We observe, first of all, that the subloop of $G$ consisting of all squares is a commutative Moufang loop. This already proves our theorem for loops all of whose elements have odd orders. One expects difficulty for loops containing elements of order two, particularly

Received by the editors October 12, 1957.

1 This formula may also be obtained from equation (3.24) of [1] by setting $L_{x}=R_{x}$. 